\title{
DESHIDRATACION Y SOBREHIDRATACION VOLUNTARIAS DURANTE EL EJERCICIO EN EL CALOR: POSIBLES FACTORES RELACIONADOS
}

\author{
${ }^{1}$ Andrea Solera Herrera $y^{1 y 2}$ Luis Fernando Aragón Vargas \\ ${ }^{1}$ Escuela de Educación Física y Deporte, Universidad de Costa Rica \\ ${ }^{2}$ Gatorade Sport Science Institute \\ Email: andrea_solera@costarricense.cr
}

\begin{abstract}
Resumen
Solera-Herrera, A. y Aragón-Vargas, L.F. (2006). Deshidratación y Sobrehidratación voluntarias durante el ejercicio en el calor: posibles factores relacionados. Revista en Ciencias del Ejercicio y la Salud 4(1), 2233. Los efectos negativos de la deshidratación sobre la salud y el rendimiento han sido bien documentados. Recientemente, también se han documentado problemas por sobrehidratación. La presente investigación se hizo para estudiar los posibles factores que influyen sobre la ingesta voluntaria de líquido durante el ejercicio y determinar si existe relación entre el grado de deshidratación voluntaria y la creencia de que la ingesta de líquido provoca cólico. Para ello, se contó con 94 estudiantes universitarios jóvenes, varones, quienes se ejercitaron a un $60 \%$ de su FCmáx durante una hora, alternando cada 10 min. entre bicicleta estacionaria, subir y bajar un escalón, y una máquina simuladora de esquí. Esto se realizó en un laboratorio de ambiente controlado a $30^{\circ} \mathrm{C}, 70 \%$ humedad relativa. Durante el ejercicio se midió la ingesta voluntaria de una bebida deportiva, así como también las percepciones de calor ambiental, llenura estomacal, malestar gastrointestinal y palatabilidad de la bebida. Los sujetos fueron pesados desnudos y secos antes y después del ejercicio. Al final se les aplicó un cuestionario sobre la importancia de la hidratación, y sus creencias sobre el cólico. El equilibrio hídrico promedio fue de $-0.14 \pm 0.98 \%$ del peso corporal, con un rango entre $-2.38 \%$ y $2.84 \%$. La tasa de sudoración promedio fue de $1208.3 \pm 381.7 \mathrm{ml} \cdot \mathrm{h}^{-1}$; y la ingesta de $1132 \pm 610 \mathrm{ml} \cdot \mathrm{h}^{-1}$. Dos análisis de regresión múltiple determinaron que: (a) los predictores más fuertes de la ingesta voluntaria de líquido fueron la importancia asignada a la hidratación durante el ejercicio $\left(\mathrm{R}^{2}=0.13 ; \mathrm{p}<0.0005\right)$ y la percepción de llenura estomacal al minuto $25\left(\mathrm{R}^{2}=0.08 ; \mathrm{p}=0.005\right)$; (b) los predictores más fuertes del equilibrio hídrico fueron la ingesta voluntaria por $\mathrm{kg}$ de peso $\left(\mathrm{R}^{2}=0.73 ; \mathrm{p}<0.0005\right)$, y la tasa de sudoración $\left(\mathrm{R}^{2}=0.08 ; \mathrm{p}=\right.$ 0.006). No obstante, el equilibrio hídrico no estuvo asociado con ninguna de las percepciones, ni con las creencias de las posibles causas de cólico. Al analizar por separado a los individuos que se deshidrataron de los que se sobrehidrataron se encontró que la sobrehidratación está más relacionada con ingerir grandes cantidades de líquido, que con tasas bajas de sudoración. PALABRAS CLAVES: Equilibrio hídrico, sobrehidratación, cólico.
\end{abstract}

\section{INTRODUCCION}

Por mucho tiempo se ha conocido el efecto negativo de la deshidratación sobre el rendimiento deportivo, pero al mismo tiempo es frecuente encontrar datos que demuestran que los practicantes de deportes incurren en una deshidratación voluntaria. Dicha deshidratación podría obedecer a distintas causas, pero la experiencia de algunos científicos versados en el tema sugiere que muchas personas físicamente activas no beben líquido antes del ejercicio ni durante éste por el temor a sufrir un cólico (Morton, Aragón-Vargas, \& Callister, 2004). A pesar de su difícil medición, el cólico es un fenómeno medible y repetible, que amerita más investigación experimental (Morton \& Callister, 2000; Morton et al., 2004). Otras posibles causas de una ingesta de líquido insuficiente podrían ser la percepción de llenura estomacal, una baja palatabilidad de la bebida, una baja percepción de calor ambiental, o la poca importancia asignada a 
la hidratación durante la actividad física en el calor (Armstrong, Hubbard, Szlyk, Matthew, \& Sils, 1985; Greenleaf \& Sargent, 1965; Hubbard et al., 1984; Passe, Horn, \& Murray, 2000; Rothstein, Adolph, \& Wills, 1947; Szlyk, Hubbard, Matthew, Armstrong, \& Kerstein, 1987; Szlyk, Sils, Francesconi, Hubbard, \& Armstrong, 1989). Por otra parte, se sabe que algunas pocas personas más bien tienen una tendencia a sobrehidratarse. Esto es particularmente importante porque si beben demasiado líquido durante el ejercicio prolongado, podrían estar en mayor riesgo de sufrir hiponatremia por dilución (Barr \& Costill, 1989; Gardner, 2002; Montain, Sawka, \& Wenger, 2001; Noakes, Goodwin, Rayner, Branken, \& Taylor, 1985).

Es claro entonces que, aunque sea deseable desde el punto de vista fisiológico mantener una buena hidratación durante la actividad física $y$ el entrenamiento y la competición deportiva, la ejecución de las recomendaciones es defectuosa, ya que algunos individuos tienden a beber demasiado $\mathrm{y}$ otros tienden a beber muy poco para sus necesidades reales; ambas situaciones tienen consecuencias negativas. La clave del éxito está en el equilibrio hídrico: beber lo suficiente para reponer lo que se pierde durante el ejercicio, ni más, ni menos.

La sobrehidratación y deshidratación voluntarias durante el ejercicio en el calor se pueden estudiar a la luz de un modelo de equilibrio hídrico según el cual el equilibrio depende de las ganancias vs. las pérdidas de agua en el cuerpo (Figura 1). En este caso, las ganancias son primordialmente por ingesta (el aporte por concepto de agua resultante del metabolismo es proporcionalmente muy pequeño). Las pérdidas son principalmente por sudoración (también puede haber producción de orina $\mathrm{y}$ heces, $\mathrm{y}$ se pierde humedad por respiración, pero las pérdidas en estas condiciones son también proporcionalmente pequeñas).

Figura 1. Modelo conceptual de equilibrio hídrico.

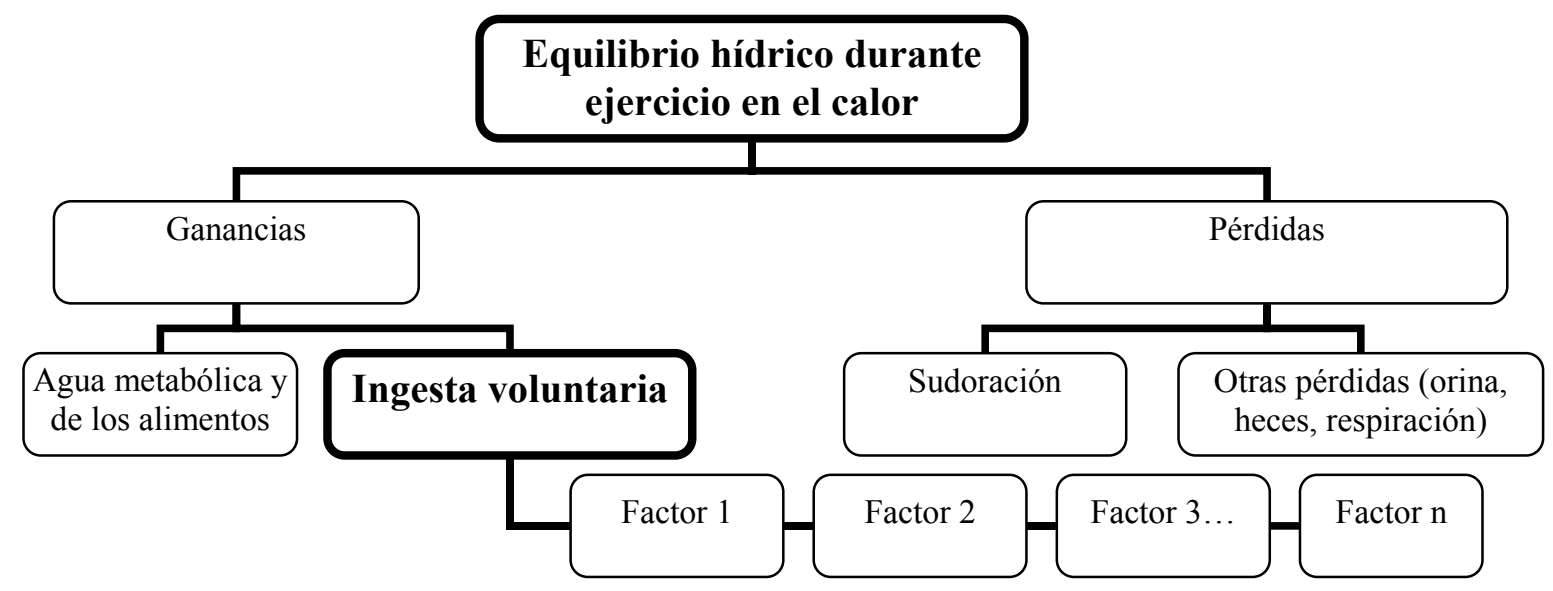

Es posible que el problema de un desequilibrio hídrico obedezca solamente a la ingesta voluntaria per se, o que obedezca a la ingesta relativa a la pérdida de líquido por sudoración. Por lo tanto, el presente estudio tuvo dos objetivos principales: En primer lugar, identificar aquellos posibles factores que expliquen la ingesta voluntaria de líquido durante el ejercicio en ambiente controlado. En segundo lugar, identificar los posibles 
factores que expliquen el grado de deshidratación o sobrehidratación voluntaria (equilibrio hídrico). Interesaba especialmente evaluar la relación de la ingesta voluntaria y del equilibrio hídrico con la percepción de que la ingesta de líquido durante el ejercicio provoca cólico

\section{METODOLOGIA}

\section{Participantes}

Se contó con la participación de 94 adultos jóvenes, varones, provenientes de los cursos universitarios de actividad deportiva, y que realizaban actividad física por lo menos 3 veces por semana. Los voluntarios dieron su consentimiento de participación conforme a las directrices establecidas por el Comité Ético Científico de la Universidad de Costa Rica, pero desconocieron el verdadero objetivo de la prueba ya que el conocimiento previo habría afectado su comportamiento y por ende los resultados obtenidos.

\section{Instrumentos de medición}

El peso corporal se midió con una báscula digital Acculab ${ }^{\circledR}$ SV-100, con una precisión de $20 \mathrm{~g}$. Los pesos pre- y postejercicio se utilizaron para calcular el equilibrio hídrico, mediante la ecuación

$$
\text { Eq.Hídrico }=\frac{P_{\text {final }}-P_{\text {inicial }}}{P_{\text {inicial }}} * 100
$$

De estos datos también se derivó la tasa de sudoración en mililitros por hora, restando el peso final al peso inicial en gramos, y sumándole la ingesta total de líquido.

Se utilizó un cuestionario de percepciones para evaluar la percepción de llenura estomacal (escala de 0 -nada- a 9 lleno a reventar-), la palatabilidad de la bebida (escala convencional de 1-muy, muy desagradable- a 9 -muy, muy agradable-), la presencia de algún malestar gastrointestinal (escala de 0 -ningún dolor- a 9 -dolor insoportable), y la percepción de temperatura ambiental (escala de 1 -frío insoportable- a 9 -calor insoportable-).

Para calcular el volumen de líquido ingerido se utilizó la sumatoria de las ingestas de cada período de 20 minutos dividido por el peso corporal inicial. Cada ingesta se calculó sustrayendo al volumen de líquido inicial, el volumen de líquido remanente de cada botella. Los volúmenes inicial y remanente en las botellas se midieron con una probeta graduada con una precisión de $1 \mathrm{~mL}$.

Se elaboró una "Encuesta sobre el cólico y sus causas", enumerando aquellas causas de cólico que son más comúnmente citadas por las personas físicamente activas, y solicitando a cada participante que asignara a cada una un valor entre 1 (totalmente en desacuerdo) y 5 (totalmente de acuerdo). Cada ítem del cuestionario fue utilizado como una posible variable predictora, pero el análisis principal de creencias se basó en dos puntajes de causas: a) Que el cólico se debe a la ingesta de líquido antes de o durante el ejercicio (sumatoria de dos ítems), y b) Que el cólico se debe a otras causas aparte de la ingesta de líquido (sumatoria de los ítems restantes).

$\mathrm{La}$ importancia asignada a la rehidratación durante el ejercicio en el calor se cuantificó mediante una pregunta al respecto en el mismo cuestionario, usando la misma escala de 1 a 5.

\section{Procedimientos}

Los participantes realizaron una hora de ejercicio a una intensidad moderada $(60 \%$ de su reserva cardiaca máxima), controlada mediante monitores de frecuencia cardiaca Polar ${ }^{\circledR}$. El ejercicio alternó cada diez minutos entre las estaciones de cicloergómetro, subir y bajar una grada de $30 \mathrm{~cm}$. de altura, y simulación de esquí a campo traviesa ("Nordic Track ${ }^{\circledR \text { ") }}$, todo en un laboratorio de ambiente controlado donde la temperatura se mantuvo a $30^{\circ} \mathrm{C}$ y la humedad relativa al $70 \%$ (índice de estrés térmico WBGT $=26.8^{\circ} \mathrm{C}$ ). Antes y después de la sesión de ejercicio, cada sujeto fue pesado desnudo y seco. 
Durante el ejercicio, los participantes tuvieron a su disposición una botella con suficiente bebida deportiva (Gatorade ${ }^{\circledR}, 6 \%$ de carbohidratos, $20 \mathrm{mEq} / \mathrm{L}$ de sodio), inicialmente a $8^{\circ} \mathrm{C}$. La botella se cambió por lo menos cada 20 minutos por una botella fresca. Los sujetos fueron informados de la presencia de dicha botella, y se les indicó que podían beber cuanto quisieran.

A los 25 y 55 minutos del ejercicio, se aplicó el cuestionario de percepciones a cada sujeto. Además, al finalizar, se pidió a cada participante que completara un cuestionario relacionado con creencias sobre el cólico y conocimientos sobre la importancia de la rehidratación durante el ejercicio en calor.

\section{Análisis Estadístico}

Se hizo un análisis con las estadísticas descriptivas básicas de todas las variables involucradas. Para ver el efecto del tiempo sobre algunas de las variables, se compararon las percepciones entre los minutos 25 y 55 mediante pruebas $t$ de Student para medidas repetidas y correlaciones. Además, se comparó la ingesta parcial cada veinte minutos mediante un análisis de varianza de una vía de medidas repetidas.

Se realizaron dos análisis de regresión múltiple, para determinar cuáles fueron las variables predictoras más fuertes de a) la ingesta voluntaria de líquido y b) el equilibrio hídrico. Como variables predictoras, se incluyeron: tasa de sudoración, percepción de llenura estomacal, palatabilidad de la bebida, percepción de calor ambiental, presencia de malestar gastrointestinal, la importancia asignada a la rehidratación durante el ejercicio en el calor y las creencias sobre las causas del cólico. Los modelos de regresión múltiple se calcularon utilizando regresión "stepwise" con 0.05 como criterio de significancia de ingreso y 0.1 como criterio de significancia de exclusión.

Finalmente, para explorar cuáles son las posibles diferencias entre aquellos participantes que se deshidrataron y aquéllos que más bien se sobrehidrataron durante la sesión de ejercicio, se definieron dos grupos extremos en forma objetiva y se realizaron pruebas $t$ de Student para muestras independientes, comparando estos dos grupos en cada una de las variables indicadas en el párrafo anterior, así como en la edad, estatura, peso corporal y nivel habitual de actividad física.

\section{RESULTADOS}

Los participantes fueron jóvenes (19.6 \pm 2.1 años) (promedio \pm desviación estándar), de peso y estaturas normales para la edad $(65.6 \pm 9.2 \mathrm{~kg}, 1.71 \pm 0.06 \mathrm{~m})$. La frecuencia cardiaca meta durante el ejercicio fue de $152.1 \pm 5.0$ latidos por minuto. Estos sujetos se podrían calificar como poco propensos al cólico (1.5 \pm 0.9 episodios en el último año), aunque la mayoría de ellos (92.5\%) dice saber lo que es este fenómeno. La mayoría de los participantes (84.9\%) acostumbra hidratarse cuando se ejercita; el grupo estudiado le da una importancia alta a la hidratación para la actividad física (puntaje de $4.2 \pm 0.9$, en escala de 1 a 5 ).

La figura 2 muestra la distribución del estado de hidratación o equilibrio hídrico al final del tiempo de ejercicio. Durante la sesión de ejercicio el promedio de deshidratación fue muy leve $(0.14 \%$ del peso corporal); sin embargo, hubo gran variabilidad en el equilibrio hídrico individual, desde un extremo de deshidratación de $-2.38 \%$ del peso corporal, hasta un individuo que terminó con una sobrehidratación de $2.84 \%$ de su peso. La ingesta de este último individuo fue extremadamente alta $(3862 \mathrm{~mL}$ en una hora de ejercicio) pero no fue eliminado de los análisis porque hubo certeza de que sus datos eran correctos, aunque claramente se trata de un puntaje extremo. Así mismo, la ingesta voluntaria de líquido varió enormemente, desde $20 \mathrm{~mL}$ hasta $2524 \mathrm{~mL}$ por hora (sin contar el sujeto mencionado anteriormente). Un sujeto sí fue eliminado de los análisis, ya que sus resultados mostraban claramente que hubo errores en la recolección de datos; la 
muestra finalmente analizada fue de 93 participantes.

La estadística descriptiva de las principales variables se puede observar en la Tabla 1. Los puntajes de percepción obtenidos entre los minutos 25 y 55, muestran un deterioro estadísticamente significativo, aunque no pronunciado (Figura 3). La percepción de llenura aumentó $(\mathrm{p}<0.0005)$, la palatabilidad de la bebida disminuyó $(\mathrm{p}=0.022)$, la percepción de malestar gastrointestinal aumentó $(\mathrm{p}=0.005)$, y la percepción de calor aumentó $(\mathrm{p}=0.001)$. También se verificaron correlaciones positivas $(p<0.05)$ entre los puntajes de percepción obtenidos al minuto $25 \mathrm{y}$ aquéllos obtenidos al minuto 55: llenura $\mathrm{r}=0.70$, palatabilidad $\mathrm{r}=0.84$, malestar gastrointestinal $\mathrm{r}=0.55$ y calor $\mathrm{r}=0.21$. Por otra parte, al desglosar la ingesta voluntaria entre los tres períodos de 20 minutos se verificó que no hubo una diferencia significativa entre éstos $(p=0.23)$. Las correlaciones de las ingestas parciales con la ingesta total fueron $\mathrm{r}=0.82$, $\mathrm{r}=0.81$ y $\mathrm{r}=0.71$ para el primer, segundo, $\mathrm{y}$ tercer períodos de veinte minutos, respectivamente $(\mathrm{p}<0.0005)$.

La Tabla 2 reporta los factores que los participantes perciben como causantes del cólico, por orden de importancia, según el puntaje total obtenido. Beber o comer antes de hacer ejercicio son percibidos como factores sumamente importantes.

Figura 2. Equilibrio hídrico o estado de hidratación de cada participante $(\mathrm{n}=93)$. Las líneas punteadas indican cuáles sujetos se clasificaron como deshidratados o sobrehidratados (ver explicación al final de los resultados).

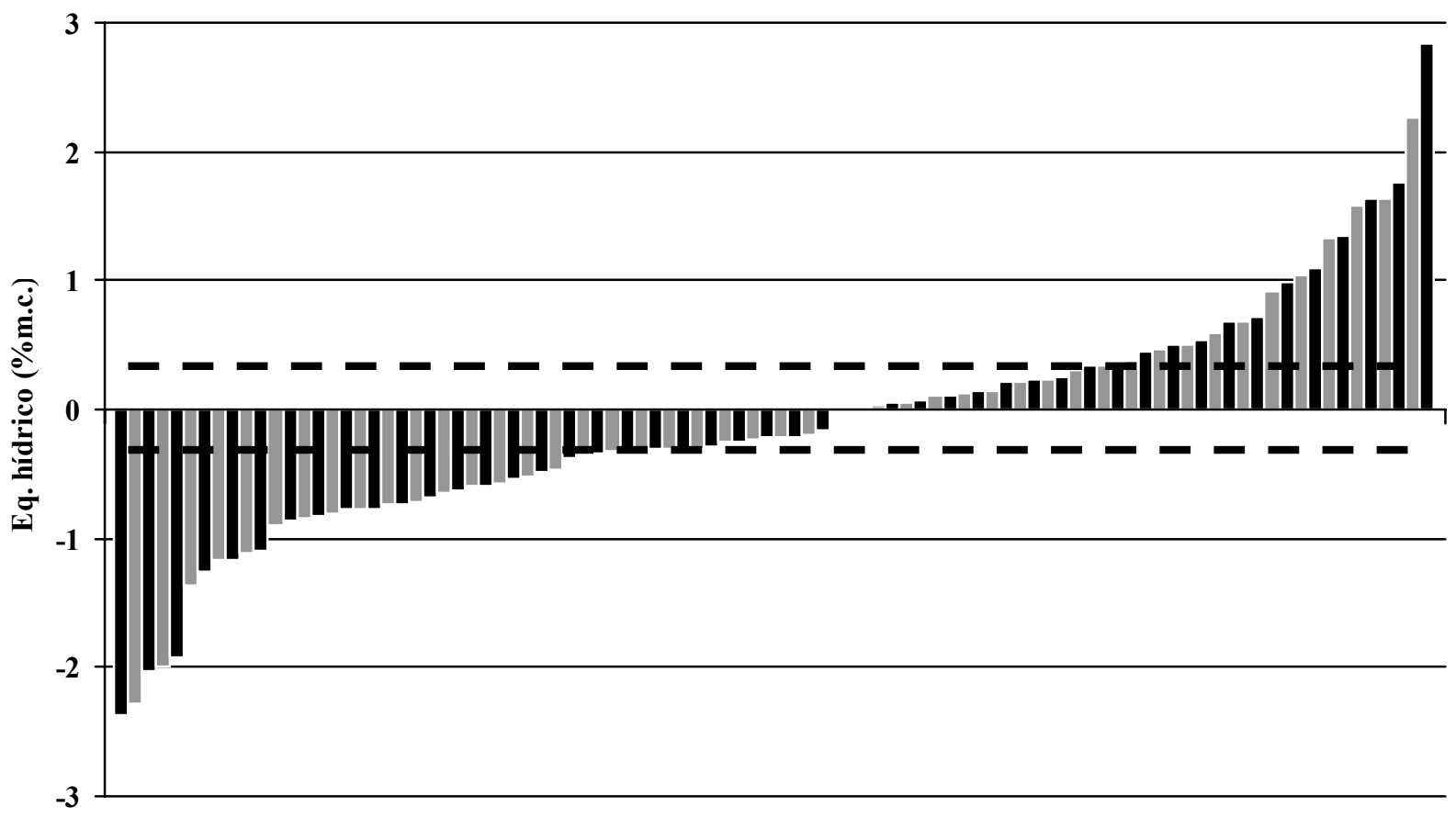


Tabla 1. Estadística descriptiva $(n=93)$

\begin{tabular}{lllll}
\hline & MIN & MAX & Promedio & SD \\
\cline { 2 - 4 } Peso inicial & 43.00 & 93.96 & 65.639 & 9.247 \\
Peso final & 43.58 & 93.20 & 65.545 & 9.215 \\
Cambio de peso & -1.98 & 1.46 & 0.094 & 0.578 \\
Ingesta del minuto 0 al 20 & 0 & 2330 & 352.1 & 320.4 \\
Ingesta del minuto 20 al 40 & 10 & 1162 & 403.8 & 200.3 \\
Ingesta del minuto 40 al 60 & 0 & 1162 & 376.5 & 258.9 \\
Ingesta total & 20 & 3862 & 1132.4 & 610.0 \\
Tasa de sudoración(mI/hora) & 102 & 2518 & 1208.3 & 381.7 \\
Equilibrio hídrico & -2.38 & 2.84 & -0.14 & 0.89 \\
Percepción de llenura (min 25) & 0 & 7 & 2.3 & 1.8 \\
Percepción de llenura (min 55) & 0 & 8 & 3.1 & 2.2 \\
Percepción palatabilidad (min 25) & 2 & 9 & 7.2 & 1.6 \\
Percepción palatabilidad (min 55) & 1 & 9 & 7.0 & 1.7 \\
Percepción de malestar GI (min 25) & 0 & 5 & 0.4 & 1.1 \\
Percepción de malestar GI (min 55) & 0 & 7 & 0.8 & 1.5 \\
Percepción del calor (min 25) & 5 & 9 & 7.0 & 0.6 \\
Percepción del calor (min 55) & 5 & 9 & 7.3 & 1.0 \\
Importancia de la rehidratación & 2 & 5 & 4.2 & 0.9 \\
Ingesta por kilogramo de peso & 0.33 & 55.39 & 17.37 & 9.16 \\
Creencia de cólico por ingesta de líquido & 3 & 10 & 6.7 & 1.7 \\
Creencia de cólico por otras razones & 16 & 30 & 22.3 & 3.3 \\
\hline
\end{tabular}

Tabla 2: Percepción de factores causantes del cólico

\begin{tabular}{|l|c|}
\hline \multicolumn{1}{|c|}{ Variable } & $\begin{array}{l}\text { Promedio } \pm \\
\text { ds }\end{array}$ \\
\hline Comer antes del ejercicio & $4.02 \pm 0.92$ \\
\hline Respirar mal & $3.87 \pm 0.96$ \\
\hline Beber antes del ejercicio & $3.71 \pm 1.04$ \\
\hline Mala condición física & $3.67 \pm 1.07$ \\
\hline $\begin{array}{l}\text { Tomar líquido durante } \\
\text { ejercicio }\end{array}$ & $2.98 \pm 1.05$ \\
\hline Mal calentamiento & $2.98 \pm 1.12$ \\
\hline Ejercicio intenso & $2.71 \pm 1.17$ \\
\hline Golpes o sacudidas & $2.57 \pm 0.93$ \\
\hline Ejercicio prolongado & $2.47 \pm 0.88$ \\
\hline
\end{tabular}

$(n=93)$ Escala: 1=Totalmente en desacuerdo a $5=$ Totalmente de acuerdo
Figura 3: Cambios en la percepción promedio entre los minutos 25 y $55(n=93)$

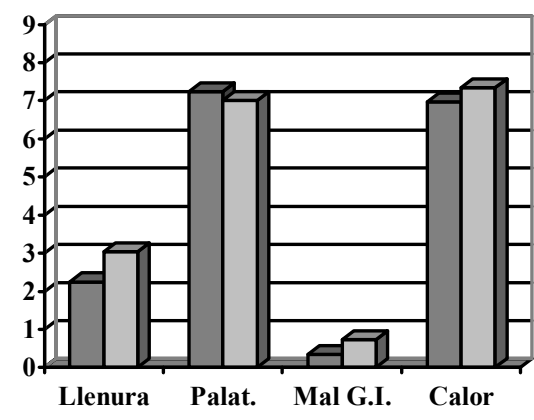

$\square$ Min. $25 \square$ Min. 55

Las cuatro variables cambian significativamente $(p<0.05)$ 


\section{Ingesta voluntaria de líquido:}

En primer lugar, no se encontró una relación significativa entre la ingesta total de líquido (bruta) y la edad, el peso, o la estatura. La Tabla 3 muestra los modelos de regresión más significativos, utilizando la ingesta por kilogramo de peso como variable dependiente. El predictor simple más relevante es la importancia asignada a la hidratación durante el ejercicio, que explica el $13 \%$ de la varianza en la ingesta voluntaria. La predicción mejora a un $17 \%$ si se añade la percepción de llenura al minuto 25 . No se encontró relación entre la ingesta voluntaria de líquido y la creencia de que la ingesta de líquido provoca cólico.

Tabla 3. Modelos de regresión con ingesta voluntaria por kilogramo de peso como variable dependiente

\begin{tabular}{lccc}
\hline Predictor (es) & $\mathbf{R}$ & $\mathbf{R}^{\mathbf{2}}$ & $\mathbf{p}$ \\
\cline { 2 - 4 } Importancia rehidratación + perc. Ilenura (25) & .417 & .174 & $<.0005$ \\
Importancia de la rehidratación & .357 & .128 & $<.0005$ \\
Percepción de llenura (55) & .292 & .085 & .004 \\
Percepción de llenura (25) & .290 & .084 & .005 \\
Tasa de sudoración & .172 & .030 & .098 \\
Percepción de malestar GI (25) & .168 & .028 & .108 \\
Creencia de que el cólico obedece a otras razones & -.061 & .004 & .564 \\
Percepción de palatabilidad (25) & .050 & .003 & .636 \\
Percepción de malestar GI (55) & .048 & .002 & .645 \\
Creencia de que ingesta de líquido provoca cólico & -.041 & .002 & .698 \\
Percepción de calor ambiental (25) & -.039 & .002 & .710 \\
Percepción de calor ambiental (55) & .002 & .000 & .981 \\
Percepción de palatabilidad (55) & -.001 & .000 & .994 \\
\hline
\end{tabular}

$(\mathrm{n}=93)$. Una correlación positiva indica que conforme aumenta el predictor, aumenta la ingesta voluntaria.

\section{Equilibrio hídrico:}

La Tabla 4 muestra los modelos de regresión más significativos, utilizando el equilibrio hídrico como variable dependiente. El predictor simple más relevante es la ingesta voluntaria por kilogramo de peso, que explica el $72.8 \%$ de la varianza en el equilibrio hídrico. La tasa de sudoración es el siguiente, pero por sí solo explica únicamente un $8 \%$ de la varianza en el estado de hidratación. Combinando ambas variables en un solo modelo, se explica $91.7 \%$ de la varianza, y añadiendo a éstas el peso inicial, un $96.5 \%$ de la varianza. El equilibrio hídrico no estuvo asociado significativamente con la percepción de malestar gastrointestinal, de palatabilidad de la bebida, ni de calor ambiental; tampoco tuvo relación con las creencias sobre el cólico ni con el nivel habitual de actividad física.

\section{Contraste entre sobrehidratados y deshidratados:}

Para subdividir al grupo se estableció objetivamente el criterio de considerar deshidratados a aquellos participantes que finalizaron con un equilibrio hídrico menor o igual a $-0.3 \% \quad(n=40)$. Igualmente, se consideraron sobrehidratados aquéllos que alcanzaron un equilibrio hídrico (grado de sobrehidratación) mayor o igual a $0.3 \%(\mathrm{n}=$ 28). Estos puntos de corte se escogieron tomando en cuenta que el esfuerzo fue relativamente corto $y$ de intensidad moderada, junto con el hecho de que ya a partir de $1.0 \%$ de deshidratación se notan efectos adversos sobre el rendimiento físico en el calor (Aragón-Vargas, Maughan, Rivera-Brown, Meyer, Murray, de Barros, García, Sarmiento, Arroyo, Javornik, 
Matsudo, Salazar \& Lentini, 2000). El resto se clasificaron como en equilibrio hídrico (n = 25) (ver fig. 1). La Tabla 5 muestra las variables que mostraron las diferencias más claras entre los grupos extremos.

Los participantes que se sobrehidrataron tuvieron ingestas parciales $y$ totales significativamente mayores que quienes se deshidrataron. La ingesta total, corregida por kilogramo de peso corporal fue más del doble. Además, su tasa de sudoración fue menor. No hubo diferencia entre grupos en cuanto a percepción de llenura, palatabilidad, malestar G.I., ni calor; edad, nivel de actividad física, creencias sobre el cólico, costumbre de rehidratarse, ni importancia asignada a la hidratación $(\mathrm{p}>0.05)$.

Tabla 4. Modelos de regresión con el equilibrio hídrico como variable dependiente

\begin{tabular}{lcc}
\hline Predictor (es) & $\mathbf{R}$ & $\mathbf{R}^{\mathbf{2}}$ \\
\hline Ingesta por kg peso - tasa sudoración + peso inicial & .982 & .965 \\
Ingesta por kg peso - tasa de sudoración & .958 & .917 \\
Ingesta voluntaria, por kilogramo de peso & .853 & .728 \\
-Tasa sudoración+percep.llenura (55)+import. rehidrata. & .210 \\
Tasa de sudoración & .459 & .0005 \\
Percepción de llenura (55) & -.283 & .080 \\
Importancia de la rehidratación & .280 & .078 \\
Percepción de llenura (25) & .267 & .071 \\
Percepción de malestar GI (25) & .209 & .044 \\
Creencia de que el cólico obedece a otras razones & .161 & .026 \\
Percepción de calor ambiental (55) & -.088 & .008 \\
Percepción de palatabilidad (25) & .074 & .005 \\
Percepción de malestar GI (55) & .040 & .005 \\
Percepción de palatabilidad (55) & .032 & .002 \\
Percepción de calor ambiental (25) & .020 & .001 \\
Creencia de que ingesta de líquido provoca cólico & .012 & .000 \\
Nivel habitual de actividad física & -.010 & .044 \\
\hline n = 93. Una correlación positiva indica que conforme aumenta el predictor, más alto es el equilibrio hídrico (menor el grado de \\
deshidratación), excepto en aquellos modelos donde los predictores son precedidos por un signo negativo (-)
\end{tabular}

Tabla 5. Comparación de promedios entre los sujetos sobrehidratados $(n=28)$ y los deshidratados $(n=40)$

\section{Ingesta por kilogramo de peso}

\section{ESTATURA}

Ingesta del minuto 0 al 20

Ingesta del minuto 20 al 40

Ingesta del minuto 40 al 60

Ingesta total

Tasa de sudoración(ml/hora)

Importancia de la rehidratación

$\begin{array}{ccccc}\text { Equilibrio hídrico } & \text { N } & \text { Media } & \text { S. D. } & \text { p-value } \\ \text { Deshidratado } & 40 & 11.70 & 6.21 & \\ \text { Sobrehidratado } & 28 & 26.37 & 9.06 & .000 \\ \text { Deshidratado } & 40 & 1.73 & .049 & \\ \text { Sobrehidratado } & 28 & 1.70 & .059 & .038 \\ \text { Deshidratado } & 40 & 273.8 & 247.4 & \\ \text { Sobrehidratado } & 28 & 556.2 & 423.9 & .001 \\ \text { Deshidratado } & 40 & 295.6 & 156.8 & \\ \text { Sobrehidratado } & 28 & 574.2 & 173.2 & .000 \\ \text { Deshidratado } & 40 & 222.4 & 178.1 & \\ \text { Sobrehidratado } & 28 & 569.8 & 235.6 & .000 \\ \text { Deshidratado } & 40 & 791.8 & 451.4 & \\ \text { Sobrehidratado } & 28 & 1700.1 & 610.7 & .000 \\ \text { Deshidratado } & 40 & 1367.3 & 415.4 & \\ \text { Sobrehidratado } & 28 & 1112.8 & 341.7 & .010 \\ \text { Deshidratado } & 40 & 4.0 & 1.0 & \\ \text { Sobrehidratado } & 28 & 4.3 & 0.8 & .129\end{array}$




\section{DISCUSION}

Los objetivos originales de este estudio eran (a) identificar aquellos posibles factores que expliquen la ingesta voluntaria de líquido durante el ejercicio en ambiente controlado, y (b) identificar los posibles factores que expliquen equilibrio hídrico. Sin embargo, se partía del supuesto que la mayoría de los sujetos sufrirían una deshidratación considerable, como se observa a menudo en condiciones de entrenamiento y competición. El hallazgo más importante de esta investigación es que aún en condiciones de estrés térmico considerable que produjeron una tasa de sudoración de aproximadamente $1200 \mathrm{~mL} / \mathrm{h}$, la deshidratación promedio fue mínima. Esto porque hubo un número considerable de participantes que se sobrehidrataron en más de $0.3 \%$ (13 sujetos se sobrehidrataron $1 \%$ o más). La tasa de sudoración obtenida no es especialmente baja: Shirreffs et al. encontraron una tasa promedio de $1462 \mathrm{~mL} / \mathrm{h}$ en jugadores profesionales de fútbol entrenando en condiciones moderadamente altas de estrés térmico (Shirreffs et al., 2005). Rivera-Brown et al. encontraron una tasa promedio de 500 $\mathrm{mL} / \mathrm{h}$ en niños varones aclimatados al calor ejercitándose en condiciones altas de estrés térmico (Rivera-Brown, Gutiérrez, Gutiérrez, Frontera, \& Bar-Or, 1999). Se muestra entonces que aún con una tasa de sudoración relativamente alta, dadas las condiciones correctas (disponibilidad de bebida de sabor y temperatura agradables), para algunos individuos sí es fácil sobrehidratarse. Esto podría traducirse en una sobrehidratación peligrosa si el patrón de ingesta de líquido se mantuviera por varias horas. Los datos de ingesta parcial y las correlaciones de las ingestas parciales con la ingesta total (todas mayores a $r=0.71$ ) sugieren que la "maña" de beber volúmenes altos se comportó como un rasgo consistente en cada individuo, y no fue el resultado de una ingesta exagerada en un momento determinado de la sesión de ejercicio.
La mayoría de los estudios publicados hasta la fecha muestran una deshidratación voluntaria promedio importante, ya sea con agua o con bebidas deportivas (American College of Sports Medicine, 1996; Calderón \& Aragón-Vargas, 1989; Shirreffs et al., 2005). Sin embargo, otros estudios con niños y jóvenes varones han demostrado que cuando se utiliza una bebida deportiva de buena palatabilidad como en este estudio, y si la bebida está altamente disponible, los niños sí beben suficiente para evitar la deshidratación (Wilk \& Bar-Or, 1996; Wilk, Kriemler, Keller, \& Bar-Or, 1998).

En lo que respecta al primer objetivo, el predictor más importante de la ingesta voluntaria fue la importancia asignada a la hidratación. Los participantes en este estudio mostraron congruencia entre esta creencia y su conducta (cosa que no siempre sucede en diversos ámbitos de la vida real). Solamente otro predictor fue significativo: la percepción de llenura (sea al minuto 25 ó 55), pero por la naturaleza de la variable, sentirse más lleno probablemente se trata de una consecuencia, más que de una causa, de una mayor ingesta. Contrario a lo esperado, la creencia de que la ingesta de líquido provoca cólico no tuvo una asociación significativa con la ingesta real. En este estudio no se observa una relación entre la palatabilidad y la ingesta voluntaria de líquido. Passe et al. (2000) han demostrado que existe una correlación positiva significativa entre estas dos variables. Sin embargo, ellos lo han demostrado usando varias bebidas distintas que dan mayor variabilidad en la palatabilidad, mientras que en el presente estudio la bebida fue una sola que tuvo calificaciones relativamente consistentes en su palatabilidad. En este caso, si se toman dos subgrupos extremos, aquellos sujetos que calificaron la bebida con 9, y aquéllos que la calificaron con 6 o menos, se obtiene una ingesta significativamente mayor en quienes dieron mejor calificación a la bebida $(21.5 \mathrm{~mL} / \mathrm{kg}$ vs. $16.4 \mathrm{~mL} / \mathrm{kg}, \mathrm{p}=$ 0.025). Otra forma de verlo es tomar el quintil superior de ingesta voluntaria $(\geq$ $1568.8 \mathrm{~mL})$ y compararlo con el quintil 
inferior $(\leq 685.2 \mathrm{~mL})$, y ver el promedio de percepción de sabor: 7.5 vs $6.9(\mathrm{p}=0.09)$ respectivamente. Excluyendo al sujeto \#24, los promedios son 7.8 vs. $6.9(\mathrm{p}=0.01)$. Esto confirmaría las observaciones de Passe et al. (2000).

Referente al segundo objetivo, por definición, el equilibrio hídrico tiene que estar fuertemente asociado con la ingesta voluntaria y con la tasa de sudoración. Sin embargo, como se observa en el cuadro \#4, la varianza explicada por cada una de estas dos variables $(73 \%$ vs. $8 \%$, respectivamente) indica que en realidad la ingesta voluntaria es un predictor muchísimo más importante del equilibrio hídrico que la tasa de sudoración. Al hacer el contraste entre sujetos sobrehidratados y deshidratados, se observó que los participantes que se sobrehidrataron tuvieron ingestas parciales y totales mayores. La ingesta total corregida por kilogramo de peso corporal fue más del doble, aunque su tasa de sudoración fue menor. Es curioso que no hubiera diferencia entre grupos en cuanto a la importancia asignada a la hidratación durante la actividad física ( $\mathrm{p}>0.05)$, ya que en el análisis de regresión ésta sí fue un predictor importante, tanto de la ingesta voluntaria de líquido como del equilibrio hídrico alcanzado durante el ejercicio. A la hora de comparar entre los sujetos sobrehidratados y los deshidratados, la prueba t no da significativa, pero debe observarse el intervalo de confianza del $95 \%$ de la diferencia entre los dos grupos, que fue entre -0.80 y 0.10 .

Así como sucedió con la ingesta voluntaria, el equilibrio hídrico no tuvo una relación significativa con la creencia de que el beber provoca cólico. Las distintas percepciones medidas en este estudio demostraron ser una guía pobre para el mantenimiento de un equilibrio hídrico adecuado. Lamentablemente, éstas no incluyeron la percepción de sed. Es posible que la sed haya estado más claramente asociada con la ingesta de líquido o con alguna otra variable como la sudoración, pero la variabilidad observada en el equilibrio hídrico de estos 93 sujetos sugiere que este mecanismo fisiológico no es lo suficientemente preciso para lograr mantener la euhidratación en seres humanos que se ejercitan durante una hora en el calor. Lo ideal es, por lo tanto, contar con una medición objetiva de las pérdidas típicas de cada individuo, para diseñar así una estrategia individualizada de ingesta de líquido.

Consideraciones estadísticas adicionales:

Todos los análisis se repitieron excluyendo al sujeto \#24, el puntaje extremo, para ver si eso cambiaba de alguna manera importante los resultados. Estos fueron básicamente los mismos que se reportaron con $\mathrm{n}=93$, con una importante excepción: eliminando a ese sujeto, la diferencia en percepción de sabor de la bebida entre quienes se sobrehidrataron y quienes se deshidrataron fue significativa a los 25 minutos $(7.7 \pm 1.1$ vs. $6.9 \pm 1.7$, respectivamente, $\mathrm{p}=0.04), \mathrm{y}$ casi significativa a los 55 minutos $(7.5 \pm 1.3$ vs. $6.8 \pm 1.8$, respectivamente, $\mathrm{p}=0.08$ ), lo cual contrasta claramente con lo obtenido anteriormente. Éste es un excelente ejemplo de los efectos que puede tener la presencia de un puntaje extremo, aún con una muestra de casi cien participantes.

El modelo de regresión obtenido más poderoso fue aquél que incluyó tanto la ingesta voluntaria como la tasa de sudoración, explicando un $96.5 \%$ de la varianza en el equilibrio hídrico. Sorprende que la varianza explicada en este caso no fuera $100 \%$, ya que por definición, el equilibrio hídrico se calculó usando el peso inicial, la ingesta, y el peso perdido. Es posible que la varianza no explicada corresponda a errores en la medición.

Al realizar análisis de regresión múltiple, se debe evaluar si existe un alto grado de colinearidad entre algunas variables, ya que esto tendrá el efecto de que si se incluye una de ellas en el modelo de regresión, las demás quedarán excluídas del modelo, aunque ello no signifique que no sean predictoras importantes por sí mismas. P.ej., la 
correlación entre la ingesta voluntaria total bruta y la ingesta por kilogramo de peso es alta $(\mathrm{r}=0.96, \mathrm{p}<0.0005)$, de manera que solamente una de ellas podrá aparecer en un mismo modelo de regresión múltiple como predictor significativo del equilibrio hídrico, aunque ambas sean importantes.

En resumen, se observó que aún ejercitándose en el calor, la disponibilidad de una bebida agradable y fresca en cantidad suficiente permite que la deshidratación promedio sea mínima, aunque algunos sujetos se sobrehidratan y otros se deshidratan considerablemente durante el ejercicio. La ingesta voluntaria de líquido tuvo como mejor predictor la creencia de que la hidratación es importante durante el ejercicio. La sobrehidratación estuvo más fuertemente asociada con el beber demasiado que con tasas bajas de sudoración; la importancia atribuida a la hidratación durante el ejercicio fue un predictor marginalmente significativo del equilibrio hídrico. Para mantener un equilibrio hídrico adecuado, los individuos deberían depender de un plan concreto de hidratación basado en su tasa de sudoración objetivamente medida más que en sus percepciones subjetivas. Se concluye además que no existe relación alguna entre las creencias acerca del cólico y la ingesta voluntaria de líquido o el equilibrio hídrico, durante el ejercicio realizado en las condiciones de este estudio.

\section{REFERENCIAS}

American College of Sports Medicine. (1996). ACSM Position Stand on Exercise and Fluid Replacement. Med Sci Sports Exerc, 28, i-vii

Aragón-Vargas L. F., Maughan R. J., Rivera-Brown A., Meyer F., Murray R., de Barros T. L., García P. R., Sarmiento J. M., Arroyo F., Javornik R., Matsudo V. K. R., Salazar W., Lentini N. (2000). Actividad física en el calor: termoregulación e hidratación en América Latina. Antología de Investigaciones. Bases de Nutrición Deportiva para el Inicio del Nuevo Milenio (pp. 9-19). Rosario, Argentina: Biosystem Servicio Educativo.

Armstrong L. E., Hubbard R. W., Szlyk P. C., Matthew W. T., \& Sils I. V. (1985). Voluntary dehydration and electrolyte losses during prolonged exercise in the heat. Aviat Space Environ Med, 56, 765-770.

Barr, S. I., \& Costill, D. L. (1989). Water: can the endurance athlete get too much of a good thing? J Am Diet Assoc, 89, 1629-1632, 1635.

Calderón M. F.,\& Aragón-Vargas L. F. (1989). Body fluid loss in Costa Rican runners during a 21$\mathrm{K}$ run. En Proceedings of the 32nd ICHPER Anniversary World Congress (pp. 387-390). Sullivan-Haberlein S \& Cordts HJ (Editores). Frostburg State University, Frostburg, Maryland, U.S.A.

Gardner, J. W. (2002). Death by water intoxication . Mil Med, 167, 432-434.

Greenleaf J. E., \& Sargent R. (1965). Voluntary dehydration in man. J Appl Physiol, 20, 719724.

Hubbard R. W., Sandick B. L., Matthew W. T., Francesconi R. P., Sampson J. B., Durkot M. J., Maller O., \& Engell D. B. (1984). Voluntary dehydration and alliesthesia for water. J Appl Physiol (Respirat Environ Exercise Physiol), 57, 868-875.

Montain, S. J., Sawka, M. N., \& Wenger, C. B. (2001). Hyponatremia associated with exercise: risk factors and pathogenesis. Exerc Sport Sci Rev, 29, 113-117.

Morton, D. P., \& Callister, R. (2000). Characteristics and etiology of exercise-related transient abdominal pain. Med Sci Sports Exerc, 32, 432-438.

Morton D. P., Aragón-Vargas L. F., \& Callister R. (2004). Effect of Ingested Fluid Composition on Exercise-related Transient Abdominal Pain. Int J Sports Nutr, 14, 197-208

Noakes T. D., Goodwin N., Rayner B. L., Branken T., \& Taylor R. K. N. (1985). Water intoxication: a possible complication during endurance exercise. Med Sci Sports Exerc, 17, 370-375.

Passe D. H., Horn M., \& Murray R. (2000). Impact of beverage acceptability on fluid intake during exercise. Appetite, 35, 219-229.

Rivera-Brown A. M., Gutiérrez R., Gutiérrez J. C., Frontera W. R., \& Bar-Or O. (1999). Drink composition, voluntary drinking, and fluid balance in exercising, trained, heatacclimatized boys. $J$ Appl Physiol, 86, 78-84.

Rothstein A., Adolph E. F. \& Wills J. H. (1947). Voluntary dehydration. Adolf EF and associates (editores) Physiology of Man in the Desert (pp. 254-270). New York: Interscience.

Shirreffs S. M., Aragón-Vargas L. F., Chamorro M., Maughan R. J., Serratosa L., \& Zachwieja J. J. (2005). The Sweating Response of Elite Professional Soccer Players to Training in the Heat. Int J Sports Med, 26, 90-95.

Szlyk P. C., Hubbard R. W., Matthew W. T., Armstrong L. E., \& Kerstein M. D. (1987). Mechanisms of voluntary dehydration among troops in the 
field. Mil Med, 152(8), 405-407.

Szlyk P. C., Sils I. V., Francesconi R. P., Hubbard R. W., \& Armstrong L. E. (1989). Effects of water temperature and flavoring on voluntary dehydration in men. Physiol Behav, 45(3), 639-647.

Wilk B., \& Bar-Or O. (1996). Effect of drink flavor and $\mathrm{NaCl}$ on voluntary drinking and hydration in boys exercising in the heat. $J$ Appl Physiol, 80(4), 1112-1117.

Wilk B., Kriemler S., Keller H., \& Bar-Or O. (1998). Consistency in preventing voluntary dehydration in boys who drink a flavored carbohydrate- $\mathrm{NaCl}$ beverage during exercise in the heat. Int J Sport Nutr, 8(1), 1-9. 